\section{YOUR VERSATILE} ENDODONTIC MATE

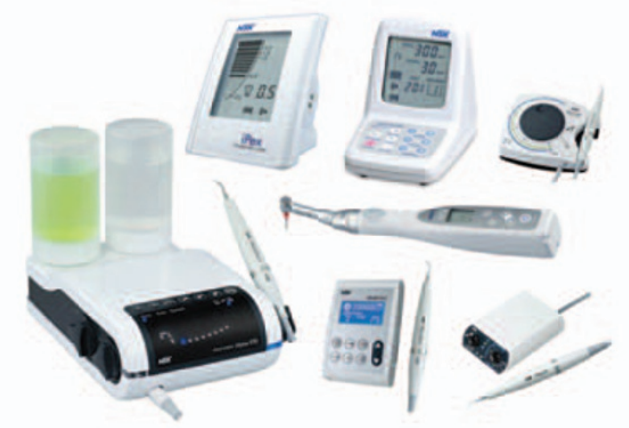

NSK's ENDO-MATE DT endodontic micromotor is specifically designed for use with Ni-Ti files from all major suppliers. This ultra-slim, lightweight endodontic micromotor delivers complete versatility with an advanced memory able to store up to nine speed and torque settings. NSK's Endo-Mate TC2 cordless handpiece has a large LCD screen incorporated in the lightweight cordless handpiece, and a simple 5 -key operation ensuring it is easy to use even during the most delicate endodontic procedures.

The NSK Varios 970 Ultrasonic scaler provides a patient-friendly and efficient way to meet the challenges of endo by exchanging the tip, and with a choice of over 70 alternative tips, there is always one to meet your needs. Each Varios 970 LUX is supplied, as standard, with a twin LED handpiece, three tips, three tip wrenches and an autoclavable container. Those looking for a more compact unit would benefit from either the Varios 570 or the Varios 370 , specifically designed as a portable control unit for easy installation into any dental unit. In addition, the Varios 170 is available as a built-in unit, where all its functions can be controlled via NSK's new MultiPad.

NSK's apex locator iPex, which accurately measures the length of any root canal, including dry, wet and bloody canals, helps clinicians perform safer, more accurate root canal treatment.

Contact NSK on 0800634 1909. www.nsk-uk.com

\title{
THE NAME'S BOND, STRONG BOND
}

Brand new FujiCEM 2 from GC is a resin-modified glass-ionomer luting cement for a wide range of indications, superseding FujiCEM.

Powered by Force \&t Fusion Technology, the enhanced FujiCEM 2 works with flexible, highly cross-linked monomers and special filler treatment which ensure improved flexural strength and higher compressive strengths to ensure an overall stronger bond. The improved retention of crowns and bridges will reward you and your patients with an even longer lasting restoration.

The two pastes of FujiCEM 2 differ in colour to clearly show that the material has been correctly mixed. The Automix of FujiCEM 2 is economical as only the material needed is extruded and mixed without substantial material excess compared with the hand mixed powder and liquid cements.

Any surplus cement can readily be removed just a minute after insertion when the material reaches a rubbery consistency. The material is not moisture-sensitive and exhibits

\section{EASILY LOCATE PRECISELY WHAT YOU WANT}

Quality Endodontic Distributors (QED) have recently launched the tenth edition of their Endodontic Specialist Catalogue.

Laid out in a very clear and concise format, with drop down style headings which mimic their online catalogue with its built-in ordering facility, it makes it extremely easy to locate and identify precisely what you want and need. continuous fluoride release together with high adhesive power and improved compressive strength. As a resin-reinforced glass-ionomer luting cement it's biocompatible and aids diagnosis due to its radiopacity. FujiCEM 2 Automix ensures safe retention of all metallic and high strength ceramic restorations and can be used for composite indirect restorations as well as all kinds of post cementation and inlays made of ceramic.

To find out more telephone GC UK on 01908 218999.
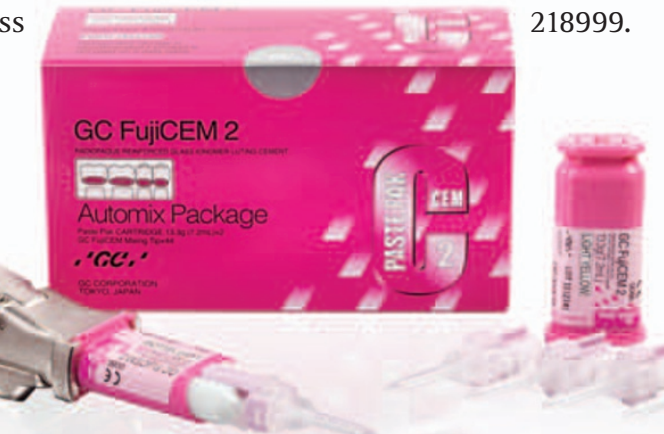